\title{
Seasonal Prediction of Marine Ecosystems: How Close Are We?
}

\author{
George Triantafyllou* \\ Hellenic Centre for Marine Research (HCMR), Institute of Oceanography, Greece
}

Submission: January 10, 2018; Published: March 23, 2018

*Corresponding author: George Triantafyllou, Hellenic Centre for Marine Research (HCMR), Institute of Oceanography, 46.7km Athens-Sounio Ave. PO Box 712, Anavyssos, Attica GR-190 13, Greece, Tel: +30-22910-76327; Fax: :+30-22910-76323; Email: gt@hcmr.gr

Abstract

The primary purpose of this article is to supply definitive statements regarding current skills in seasonal prediction with emphasis on marine ecosystem indicators and how forecasts could be possibly used for societal benefit. To enhance skills and setting priorities toward the further development and application of the existing dynamical models for seasonal prediction one has to recognize that this kind of process necessarily requires robust interactions amongst the biophysical science and applications communities and a delicate balance between scientific feasibility and application requirements. Also, recommendations for improving seasonal prediction skills and enhancing the use of seasonal prediction information for applications are outlined.

\section{Introduction}

A marine ecosystem is a complex nonlinear dynamical system, with significant spatial variability, strongly linked to the circulation of the atmosphere and oceans, and temporal variability ranging from hours to decades. Marine ecosystem forecasters interact with society mainly because of the latter's demands for accurate and detailed ocean environmental forecasts. The complexity concerning the oceanic ecosystem demonstrates that quantitative predictions can only be made using comprehensive numerical models, which encode the relevant laws of dynamics, thermodynamics and biochemistry for a complex dynamical system. Typically, such models include some millions of scalar equations, describing the interaction of physics and biochemistry on scales ranging from tens of kilometres to tens of hundreds of kilometres. These equations can only be solved on large computers.

\section{Predictability - Theory and Models}

The maximum predictability of a marine ecosystem is yet to be achieved in operational seasonal forecasting. This positioning is based upon the recognition that the model error continues to limit forecast quality and that the interactions among the nonlinear terms of the ecosystem set the limits of predictability. The fact that model error continues to be problematic is evident from the need for successful calibration efforts and the efficient use of empirical techniques to improve dynamical model forecasts. Essentially there is untapped predictability since that we currently may not take into consideration important interactions among the physical and biological components of the ecosystem.
The maximum achievable predictability is unknown and assessing this limit requires much additional research.

Chaos theory developed in an attempt to demonstrate the limited predictability of atmospheric variations [1]. In the past, the topic of predictability has been a somewhat theoretical and idealized one and to some extent not used in the practicalities of prediction. The predictability problem could well be formulated e.g. through a Liouville equation [2]. However, in practice, estimates of predictability are created from multiple (ensemble) forecasts of comprehensive atmospheric and ocean prediction models [3-6]. The uncertainty mainly in model error and initialization is quantified by contracting ensemble members, where the individual members of the ensemble differ by small perturbations [7]. For instance, the predictability of weather is largely determined by uncertainty in a forecast's starting conditions $[8,9]$, whilst the predictability of ecosystem variations is also influenced by uncertainty in representing computationally the equations that govern the biogeochemical model (for example, to what extent the phytoplankton species should be represented, and subsequently what will be the model error). Furthermore, chaos theory shows that these kinds of environmental forecasts have to be expressed probabilistically [7]; the laws of physics dictate that relatively long-term accurate weather and ocean forecasts cannot be expected. These probability forecasts quantify uncertainty in weather and ecosystem prediction. The forecaster has to strive to estimate reliable probabilities, not to disseminate predictions with a precision that cannot be justified scientifically. Examples 
have shown that, in practice, the economic value of a reliable probability forecast (produced from an ensemble prediction system) exceeds the value associated with a single deterministic forecast with uncertain accuracy $[10,11]$.

Scientific indications suggest unequivocally that predictions should be provided only as probabilities, utilizing either ensembles with dynamical models or appropriate alternatives along with empirical models. Metrics involved with probability estimations and their interpretations, are more complex compared to the deterministic ones. The necessity of a procedure that in fact sets a framework including all aspects of seasonal ecosystem forecast is incontestable considering the steadily expanding demand and use of model estimations. The purpose of hind-casts will be the standard method for improving the sample measurements. However, hind-casts may also have many challenging concerns that should be tackled, like insufficient initialization data, non-stationary nature of observing systems and non-stationary nature of the marine ecosystem.

Producing reliable probability forecasts from ensembles of atmospheric/biophysical model integrations put enormous demands on computer resources. Computer power is essential so that one can resolve the details of such a system [12]. It has been argued that, as a result of the nonlinear nature of a given system, systematic mistreatment of small-scale phenomena may lead to the systematic mistreatment of large-scale phenomena. However, one can find reasons for studying small-scale phenomena in their right. From an ecosystem prediction perspective, it is essential to have the ability to simulate such details, if models are able to forecast significant events like spring-blooms or fish stocks. However, this poses a significant dilemma given the current computing resources. To simulate extreme events, models with considerable complexity and resolution are required. On the other hand, estimating changes reliably to the probability distributions of extreme and relatively rare occasions, a large number of ensembles have to be used. One fact is inevitable; the more the necessity to provide reliable forecasts of uncertainty in the predictions of weather and ecosystem, the more the demand for computer power exceeds availability. Indeed the call for quantitative predictions of uncertainty is typically a pertinent consideration related to the design of future generations of supercomputers; ensemble prediction generally is a perfect application for parallel computing.

Model errors, particularly in areas exhibiting strong interannual variability of the ocean circulation and vertical mixing, such as the winter-spring period, continue to hamper seasonal prediction skill. The benefit of reducing the model error has not been overstated. There is a range of strategies for improving models including better representation of the interactions among the elements of the ecosystem (tuning and customization to reduce any model bias), better description of biogeochemical cycles, and substantially increased spatial resolution. All of these strategies ought to be vigorously pursued. Except the model errors, the production of seasonal forecast quality remains hindered using a wide range of factors, including:

I. Scarcity: Unlike daily forecasts, seasonal predictions can be obtained only a few times per year, creating difficulties in accumulating the information required to provide stable estimates of great quality (together with the expense of producing the predictions when ensembles are utilized),

II. Changes in variability: Predictability of ecosystem variables varies between years, across the Mediterranean, Atlantic, Baltic and Black Sea with strong evidence that these variations are also related to atmospheric and hydrodynamic variability,

III. Seasonal quality: Most metrics of seasonal forecast quality, are mainly technically presented in a nature not easily communicated to audiences outside the seasonal forecasting community.

Several broad qualitative and quantitative outcomes have been produced from the experiments recommending that seasonal predictions are more skilful in specified regions. Furthermore, climatology, or persisting recent seasonal anomalies in many cases can provide useful information. The quality of seasonal predictions varies on an inter-annual basis, partly connected to inter-annual variability of the specific marine ecosystem dynamics; average quality also differs between particular seasons. The primary seasonal variables of great interest, predictions of specific hydrodynamic variables (temperature, salinity, etc.) usually are of better quality than biogeochemical variables, such as chlorophyll, nutrients, primary production, etc.

\section{Uncertainty and Recommendations - Decision-Making Issues, Societal Benefits}

An overall recommendation, but with exceptions, is that initial conditions provided by data assimilation and used for the initialization of seasonal forecasts, improve the model projection but information on spatial patterns is lost quite quickly. It is demonstrated that in some regions and during some seasons, seasonal predictions have quality, but their translation into useful information for end-users is far from optimal; a concerted effort is essential to engage customers and seek their quantitative definition of value, so that the forecasts will be able to be used in decision-making issues. Since the direct connection between seasonal forecast quality and value has not been established, appropriate processes need to be engaged in measuring value in specific decision making instances independently from the assessment of quality. It is worth noted that a better experience in climate variability (e.g., the chances of varied scenarios, despite forecasts) could aid applications/ planning/management. The applying of forecasts requires trust in the overall quality of the forecasts and knowledge of forecast uncertainty. 
Forecast initialization is an area that requires active research. Ocean data assimilation has improved forecast quality; however, coupled data assimilation can be a field of active research that is seeking enhanced support and maybe international coordination. There is certainly significant evidence that coupled atmosphereocean-biochemical data assimilation should improve forecast quality. Multi-model methodologies $[8,13,14]$ are indeed a useful and practical approach for quantifying forecast uncertainty as a consequence of the model formulation. Still, there are open questions associated with the multi-model approach. For example, the approach is ad-hoc meaning that the choice of models isn't optimized. Nor has the community converged to any best strategy for combining the models. Multi-model calibration activities tend to yield positive results, but considerable work needs to be done, and additional research is required. The multimodel approach should not be utilized to prevent the need to improve models.

Validation has to be undertaken routinely on seasonal dynamical application models. These models ought to be complex enough to capture non-linear interactions, and at the same time being sufficiently simple to avoid over-tuning through non-constrained parameters. The relationship of forecast quality, such as applications of fish models linked to meteorological, biophysical models, is often highly non-linear. Consequently, quality of the prediction of seasonal chlorophyll might not be translated into quality within the prediction of mean fish stock, for example. Such application models like fish models should have additional metrics of forecast quality. Furthermore, these metrics should be suitable for a selected user group.

A clear need to provide information at local scales (space and time) is needed by many applications. Further effort is essential to provide and increase such information, e.g., through statistical and/or dynamical downscaling. Although there are numerous examples of seasonal forecast application (e.g., atmosphere, ocean, water management), there is potential to do considerably more. More progress has to be achieved in bringing seasonal prediction providers and seasonal prediction users together. More effort is required for the development, the production and understanding of probabilistic forecasts. More understanding of what exactly is predictable and what is not. The value of predicting 'extremes' can also be incredibly important.

The societal benefit is not fully realized, partly because there haven't been adequate interactions among physical scientists associated with seasonal prediction research and production, applications scientists, decision-makers and operational seasonal prediction providers. The issues and problems go beyond merely improving forecast quality and making forecasts readily available. The physical scientists should actively facilitate and understand users' requirements, to provide enhanced climate information, prediction products and services leading to enhanced applications. Users also have to maintain an active dialogue with the physical scientists and forecast providers so that their climate information needs are taken into account. Decision makers should take into account the seasonal forecast information, however, we ought to be aware that our products aren't the only factor they consider within the decision process. Successfully communicating uncertainty and the limitations of seasonal forecasts are essential to the process of making seasonal forecasts useful. It is challenging for several users to make explicit utilization of seasonal forecasts, most importantly when they are not following the needs of many decision makers. In several occasions, our best forecast may simply be climatology that could be useful information. Access to hind-cast data is also essential to assist users in assessing the model performance and the potential benefit from the forecasts. In these data, there is often quantitative information, which users will try to convert into terms that more closely meet their needs. Similar issues can arise regarding the spatial scale of forecast information in which the resolution of regional ecosystem models is considered too coarse for managers or decision makers. Seriously misleading situations can take place when users take information appropriate for the large scale and apply it to local scales without considering the additional uncertainty connected with such action. Application models can be used with seasonal forecasts to provide a metric that combines quality and value. For example, the quality of a seasonal forecast of fish stocks is both a proper measure of skill and a measure of potential value. Seasonal predictability research should be encouraged. Collaborations and interactions with the climate change community need to be supported and have the potential for significant benefits. Indeed, a seasonal prediction is expected to be addressed in the context of a changing climate.

\section{Acknowledgment}

This work was supported by the EU OPEC project, funded from the European Union's Seventh Framework Program (FP7/ 2007-2013) under grant agreement $n^{\circ}$ 283291. We thank Kostas Tsiaras for his kind scientific support

\section{References}

1. Lorenz EN (1963) Deterministic Nonperiodic Flow. Journal of the Atmospheric Sciences 20(2): 130-141.

2. Epstein ES (1969) Stochastic Dynamic Prediction. Tellus 21(6): 739759.

3. Toth Z, Kalnay E (1993) Ensemble forecasting at NMC: the generation of perturbations. Bulletin of the American Meteorological Society 74: 2317-2330.

4. Kalnay E, Kanamitsu M, Kistler R, Collins W, Deaven D, et al. (1996) The NCEP/NCAR 40-year reanalysis project. Bulletin of the American Meteorological Society 77(3): 437-471.

5. Toth Z, Kalnay E (1997) Ensemble forecasting at NCEP the breeding method. Monthly Weather Review 125: 3297-3318.

6. Kalnay E (2003) Atmospheric Modeling, Data Assimilation and Predictability, Cambridge, USA.

7. Leith CE (1973) The standard error of time-average estimates of climatic means. Journal of Applied Meteorology 12: 1066-1069. 
8. Buizza RA, Hollingsworth E, Lalaurette, Ghelli A (1999) Probabilistic predictions of precipitation using the ECMWF ensemble prediction system. Weather and Forecasting 14(2): 168-189.

9. Buizza R, Miller M, Palmer TN (1999) Stochastic representation of model uncertainties in the ECMWF Ensemble Prediction System. Quarterly Journal of the Royal Meteorological Society 125(560): 2887 2908.

10. Katz RW, Murphy AH (1997) Forecast value prototype decision-making models Economic value of weather and climate forecasts. Cambridge, Cambridge University Press, USA, pp. 222.
11. Palmer TN (2002) The economic value of ensemble forecasts as a tool for risk assessment: from days to decades. Quarterly Journal of the Royal Meteorological Society 128(581): 747-774.

12. Palmer TN (2000) Predicting uncertainty in forecasts of weather and climate. Reports on Progress in Physics 63(2): 71-116.

13. Ehrendorfer M (1997) Predicting the uncertainty of numerical weather forecasts: a review. Meteorol Zeitschrift 6: 147-183.

14. Houtekamer PL, Lefaivre L, Derome J, Ritchie H, Mitchell HL (1996) A system simulation approach to ensemble prediction. Monthly Weather Review 124(6): 1225-1242.

\section{Your next submission with Juniper Publishers will reach you the below assets}

- Quality Editorial service

- Swift Peer Review

- Reprints availability

- E-prints Service

- Manuscript Podcast for convenient understanding

- Global attainment for your research

- Manuscript accessibility in different formats ( Pdf, E-pub, Full Text, Audio)

- Unceasing customer service

Track the below URL for one-step submission https://juniperpublishers.com/online-submission.php 